\title{
Shape-Persistent Macrocycles with Intraannular Polar Groups: Synthesis, Liquid Crystallinity and 2D Organization
}

\section{Supporting information}

Matthias Fischer, ${ }^{\dagger}$ Günter Lieser, ${ }^{* \dagger}$ Almut Rapp, ${ }^{\dagger}$ Ingo Schnell, ${ }^{* \dagger}{ }^{\dagger}$ Wael Mamdouh, ${ }^{\ddagger}$ Steven De Feyter, ${ }^{*},{ }^{\ddagger}$ Frans C. De Schryver, ${ }^{\ddagger}$ and Sigurd Höger $*, \dagger, \S$

Max Planck Institute for Polymer Research, Ackermannweg 10, 55128 Mainz, Germany

Katholieke Universiteit Leuven (K.U.Leuven), Department of Chemistry, Celestijnenlaan 200 F, B-3001 Leuven, Belgium

E-mail: hoeger@chemie.uni-karlsruhe.de

${ }^{\dagger}$ Max Planck Institute for Polymer Research

* Katholieke Universiteit Leuven (K.U.Leuven)

$\S$ New address: Polymer Institute, Universität Karlsruhe, Hertzstr. 16, 76187 Karlsruhe, Germany 


\section{Experimental Section}

\section{General remarks.}

Reactions requiring an inert gas atmosphere were conducted under argon, and the glassware was oven-dried $\left(140^{\circ} \mathrm{C}\right)$. THF was distilled from potassium prior to use. Triethylamine, piperidine and pyridine were distilled over $\mathrm{CaH}_{2}$ and stored under argon. Commercially available chemicals were used as received. Compounds 6 and 9 are described elsewhere. ${ }^{13}{ }^{1} \mathrm{H}$ NMR and ${ }^{13} \mathrm{C}$ NMR solution spectra were recorded on a Bruker DPX 250 or AC 300 spectrometer $\left(250\right.$ and $300 \mathrm{MHz}$ for ${ }^{1} \mathrm{H}, 62.5$ and 75.48 $\mathrm{MHz}$ for ${ }^{13} \mathrm{C}$ ). Chemical shifts are given in $\mathrm{ppm}$, referenced to residual proton resonances of the solvents. Thin-layer chromatography was performed on aluminum plates precoated with Merck 5735 silica gel $60 \mathrm{~F}_{254}$. Column chromatography was performed with Merck silica gel $60(230 \pm 400$ mesh). The gel permeation chromatograms were measured in THF (flow rate $1 \mathrm{~mL} \mathrm{~min}^{-1}$ ) at room temperature, using a combination of three styragel columns (porosity $10^{3}, 10^{5}, 10^{6}$ ) and an UV detector operating at $\lambda=254 \mathrm{~nm}$. The molecular weight was obtained from polystyrene calibrated SEC columns. The matrix-assisted laser desorption ionization time-of-flight (MALDI-TOF) mass spectroscopy measurements were carried out on a Bruker reflex spectrometer (Bruker, Bremen), which incorporates a $337 \mathrm{~nm}$ nitrogen laser with a $3 \mathrm{~ns}$ pulse duration $\left(10^{6} \pm 10^{7} \mathrm{~W} / \mathrm{cm}, 100 \mathrm{~mm}\right.$ spot diameter). The instrument was operated in a linear mode with an accelerating potential of $33.65 \mathrm{kV}$. The mass scale was calibrated with polystyrene $\left(M_{p}=2300\right)$, using a number of resolved oligomers. Samples were prepared by dissolving the macrocycle in THF at a concentration of $10^{-4} \mathrm{~mol} / \mathrm{l}$. In all cases, 1,8,9-trihydroxyanthracene (Aldrich, Steinheim) was used as matrix. In general, silver trifluoroacetate was added to the samples. Field desorption spectra were recorded on a VG ZAB 2-SE FPD machine. Temperature dependent X-ray scattering was mainly performed on a home made 2circle diffractometer (Huber) in transmission mode. The diffractometer was equipped with a germanium monochromator enabling the separation of the copper $\mathrm{K}_{\alpha}$ dublett, using for the measurement $\mathrm{CuK}_{\alpha l}$ with $\lambda=1.5406 \AA$. For data recording a position sensitive $120^{\circ}$ Inel-detector was used. Electron diffraction of the solid compound was performed performed in a LEO 912 transmission electron microscope with an integrated electron energy loss spectrometer at a high voltage of $120 \mathrm{kV}$. Thin films were prepared from solution and transferred to carbon coated supporting grids prior to be molten and subsequently recrystallized on the carbon film (electron diffraction on films in the liquid crystalline state was not possible owing to the very high irradiation damage at elevated temperatures). Melting points were measured with a Reichert hot stage apparatus and are uncorrected. DSC measurements were performed at a Mettler Toledo DSC 30. Heating and cooling rates were $10^{\circ} \mathrm{C} / \mathrm{min}$. The temperatures for the crystalline-liquid crystalline transitions were read at the maximum of the peaks of the DSC trace. Microanalyses were performed by the University of Mainz. Solid-state NMR data were collected on a Bruker DRX spectrometer with a $16.4 \mathrm{~T}$ magnet (700 and $176 \mathrm{MHz}$ for ${ }^{1} \mathrm{H}$ and ${ }^{13} \mathrm{C}$, respectively). A double resonance MAS (Magic-Angle Spinning) probe was used, allowing MAS frequencies of $30 \mathrm{kHz}$. The samples $(\sim 15 \mathrm{mg})$ were packed into rotors of $2.5 \mathrm{~mm}$ outer diameter. The spectra are referenced to adamantane (1.63 ppm for ${ }^{1} \mathrm{H}$, and $38.5 \mathrm{ppm}$ for $\left.{ }^{13} \mathrm{CH}\right)$. STM experiments were performed using a Discoverer scanning tunneling microscope (Topometrix Inc. Santa Barbara, CA) with a typical frame acquisition time of $7 \mathrm{~s}$, along with an external pulse/function generator (model HP $8111 \mathrm{~A}$ ), with negative 
sample bias. Tips were etched electrochemically from Pt/Ir wire $(80 \% / 20 \%$, diameter $0.2 \mathrm{~mm}$ ) in a $2 \mathrm{~N} \mathrm{KOH} / 6 \mathrm{~N} \mathrm{NaCN}$ solution in water. Prior to imaging, the compound under investigation was dissolved in 1-phenyloctane or 1,2,4-trichlorobenzene (Aldrich) and a drop of this solution $(0.1 \mathrm{wt} \%)$ was applied on a freshly cleaved surface of highly oriented pyrolytic graphite (HOPG). The STM investigations were then performed at the liquid-solid interface. The presented STM images were acquired in the variable current mode (constant height) under ambient conditions. In the STM images, white corresponds to the highest and black to the lowest measured tunneling current. The experiments were repeated in several sessions using different tips to check the reproducibility and to avoid artefacts. After registration of an STM image of the monolayer structure, the underlying graphite lattice was recorded at the same position by decreasing the bias voltage, serving as an in situ calibration. All STM images contain raw data and are not subjected to any manipulation or image processing.

\section{Methyl-4-(2,6-diiodo-4-methyl)-phenoxybutyrate (5):}

4-Methyl-2,6-diiodophenol (4) (7.90 g , $22.0 \mathrm{mmol})$, 4-bromtrimethyl-orthobutyrate $(5.0 \mathrm{~g}, 22.0 \mathrm{mmol}), \mathrm{K}_{2} \mathrm{CO}_{3}(3.0 \mathrm{~g}, 21.7 \mathrm{mmol})$ and $\mathrm{KI}(50 \mathrm{mg})$ were stirred in DMF $(35 \mathrm{~mL})$ at $60{ }^{\circ} \mathrm{C}$ for $48 \mathrm{~h}$ and after cooling to room temperature poured into ether and water. The organic phase was separated and extracted with water, $10 \%$ acetic acid, water, $10 \%$ aqueous sodium hydroxide, water, and brine. After drying over $\mathrm{MgSO}_{4}$ and evaporation of the solvent the crude product was chromatographed over silica gel with $\mathrm{CH}_{2} \mathrm{Cl}_{2} /$ petroleum ether $(1: 4)$ as the eluent $\left(R_{\mathrm{f}}=0.45\right)$ to give $\mathbf{5}$ as a white solid $(8.09 \mathrm{~g}, 73 \%)$. Although the ortho ester hydrolyses during the workup procedure, the crude product may contain variable minor amounts of the corresponding orthoester product, seeing by an extra NMR signal at $3.19 \mathrm{ppm}$ (s). In that case the crude product was dissolved in $\mathrm{CH}_{2} \mathrm{Cl}_{2}$ and extracted several times with $10 \%$ acetic acid, then water, $10 \%$ aqueous sodium hydroxide, water, and brine to complete the transformation of the orthoester to 3. M.p. $177^{\circ} \mathrm{C} ;{ }^{1} \mathrm{H}-\mathrm{NMR}\left(300 \mathrm{MHz}, \mathrm{CD}_{2} \mathrm{Cl}_{2}\right): \delta=$ $7.60(\mathrm{~s}, 2 \mathrm{H}), 3.96(\mathrm{t}, J=5.7 \mathrm{~Hz}, 2 \mathrm{H}), 3.67(\mathrm{~s}, 3 \mathrm{H}), 2.66(\mathrm{t}, J=7.6 \mathrm{~Hz}, 2 \mathrm{H}), 2.23(\mathrm{~s}$, $3 \mathrm{H}), 2.12-2.22(\mathrm{~m}, 2 \mathrm{H}) ;{ }^{13} \mathrm{C}-\mathrm{NMR}\left(75 \mathrm{MHz}, \mathrm{CD}_{2} \mathrm{Cl}_{2}\right): \delta=173.88,155.81,140.77$, 138.37, 90.60, 72.32, 51.82, 30.98, 25.78, 19.75; elemental analysis for $\mathrm{C}_{12} \mathrm{H}_{14} \mathrm{I}_{2} \mathrm{O}_{3}$ (460.05): calcd C 31.33, H 3.07; found C 31.02, H 3.14; MS (FD): 459.9 ( $\mathrm{M}^{+}$), 920.1 $\left(2 \mathrm{M}^{+}\right)$.

\section{Methyl-4-(2,6-bis-\{2-[2,5-dipropyloxy-4-(2-triisopropylsilylethynyl)phenyl]-} ethynyl $\}$-4-methyl)-phenoxybutyrate (7): $\mathrm{Pd}\left(\mathrm{PPh}_{3}\right)_{2} \mathrm{Cl}_{2}(65 \mathrm{mg})$ and $\mathrm{CuI}(33 \mathrm{mg})$ were added to a solution of $5(1.67 \mathrm{~g}, 3.63 \mathrm{mmol})$ and $6(3.07 \mathrm{~g}, 7.70 \mathrm{mmol})$ in triethylamine/THF $(10: 1)(110 \mathrm{~mL})$. The solution was stirred for $24 \mathrm{~h}$ at room temperature, for $1 \mathrm{~h}$ heated to $50{ }^{\circ} \mathrm{C}$ and after cooling to room temperature poured into ether and water. The organic phase was separated and extracted with water, $10 \%$ acetic acid, water, $10 \%$ aqueous sodium hydroxide, water, and brine. After drying over $\mathrm{MgSO}_{4}$ and evaporation of the solvent the crude product was chromatographed over silica gel with $\mathrm{CH}_{2} \mathrm{Cl}_{2}$ /petroleum ether (slow polarity increase from 1:4 to 1:1) as the eluent $\left(R_{\mathrm{f}}\left(\mathrm{CH}_{2} \mathrm{Cl}_{2} /\right.\right.$ petroleum ether $\left.\left.1: 1\right)=0.86\right)$ to give 7 as a slightly yellow solid (3.18 g, $87 \%$ ). ${ }^{1} \mathrm{H}-\mathrm{NMR}\left(300 \mathrm{MHz}, \mathrm{CD}_{2} \mathrm{Cl}_{2}\right.$ ): $\delta=7.29$ (s, $\left.2 \mathrm{H}\right), 6.99$ (s, $\left.2 \mathrm{H}\right), 6.96$ (s, $2 \mathrm{H}), 4.36(\mathrm{t}, J=6.1 \mathrm{~Hz}, 2 \mathrm{H}), 3.96(\mathrm{~m}, 8 \mathrm{H}), 3.53(\mathrm{~s}, 3 \mathrm{H}), 2.65(\mathrm{t}, J=7.6 \mathrm{~Hz}, 2 \mathrm{H})$, $2.30(\mathrm{~s}, 3 \mathrm{H}), 2.11(\mathrm{~m}, 2 \mathrm{H}), 1.82(\mathrm{~m}, 8 \mathrm{H}), 1.15(\mathrm{~s}, 42 \mathrm{H}), 1.06(\mathrm{~m}, 12 \mathrm{H}) ;{ }^{13} \mathrm{C}-\mathrm{NMR}$ $\left(75 \mathrm{MHz}, \mathrm{CD}_{2} \mathrm{Cl}_{2}\right): \delta=173.94,158.79,154.73,153.86,134.62,133.65,117.90$, $117.16,114.67,114.37,103.46,97.05,91.19,90.61,73.73,71.62,71.44,51.58$, 
$31.15,26.15,23.14,20.52,18.88,11.83,10.79,10.68$; elemental analysis for $\mathrm{C}_{62} \mathrm{H}_{88} \mathrm{O}_{7} \mathrm{Si}_{2}$ (1001.56): calcd $\mathrm{C} 74.35, \mathrm{H}$ 8.86; found $\mathrm{C} 74.52 \mathrm{H}$ 8.99; MS (FD): $1000.5\left(\mathrm{M}^{+}\right)$.

Methyl-4-\{2,6-bis-[2-(2,5-dipropyloxy-4-ethynylphenyl)-ethynyl]-4-methyl\}phenoxybutyrate (8): A $1 \mathrm{M}$ solution of $\mathrm{Bu}_{4} \mathrm{NF}$ in THF $(12.5 \mathrm{~mL}, 12.5 \mathrm{mmol})$ was added to a solution of $7(3.15 \mathrm{~g}, 3.15 \mathrm{mmol})$ in THF $(20 \mathrm{~mL})$ and water $(0.5 \mathrm{~mL})$. The mixture was stirred for $2 \mathrm{~h}$ at room temperature and then poured into ether and water. The organic layer was extracted with water and brine and dried over $\mathrm{MgSO}_{4}$. After evaporation of the solvent the residue was purified by chromatography over silica gel with $\mathrm{CH}_{2} \mathrm{Cl}_{2}$ /petroleum ether(1:1) as the eluent and subsequently recrystallized from methanol $(20 \mathrm{~mL})$ to give 8 as a slightly yellow solid $(1.56 \mathrm{~g}, 74 \%)\left(R_{\mathrm{f}}\right.$ $\left(\mathrm{CH}_{2} \mathrm{Cl}_{2}\right.$ petroleum ether(2:3)) =0.65). M. p. $149-152{ }^{\circ} \mathrm{C} ;{ }^{1} \mathrm{H}-\mathrm{NMR}(250 \mathrm{MHz}$, $\left.\mathrm{CD}_{2} \mathrm{Cl}_{2}\right): \delta=7.30(\mathrm{~s}, 2 \mathrm{H}), 7.03(\mathrm{~s}, 2 \mathrm{H}), 7.00(\mathrm{~s}, 2 \mathrm{H}), 4.37(\mathrm{t}, J=5.7 \mathrm{~Hz}, 2 \mathrm{H}), 3.98(\mathrm{t}$, $J=6.5 \mathrm{~Hz}, 4 \mathrm{H}), 3.97(\mathrm{t}, J=6.5 \mathrm{~Hz}, 4 \mathrm{H}), 3.53(\mathrm{~s}, 3 \mathrm{H}), 3.40(\mathrm{~s}, 2 \mathrm{H}), 2.66(\mathrm{t}, J=$ $7.6 \mathrm{~Hz}, 2 \mathrm{H}), 2.31(\mathrm{~s}, 3 \mathrm{H}), 2.06-2.17(\mathrm{~m}, 2 \mathrm{H}), 1.76-1.92(\mathrm{~m}, 8 \mathrm{H}), 1.07(\mathrm{t}, J=7.5 \mathrm{~Hz}$, $4 \mathrm{H}), 1.06(\mathrm{t}, J=7.4 \mathrm{~Hz}, 4 \mathrm{H}) ;{ }^{13} \mathrm{C}-\mathrm{NMR}\left(75 \mathrm{MHz}, \mathrm{CD}_{2} \mathrm{Cl}_{2}\right): \delta=173.93,158.82$, $154.59,153.88,134.72,133.67,118.14,117.76,117.48,114.91,113.21,91.31,90.33$, 82.64, 80.36, 73.73, 71.54, 51.58, 32.67, 31.12, 26.12, 23.04, 20.52, 10.64; elemental analysis for $\mathrm{C}_{44} \mathrm{H}_{48} \mathrm{O}_{7}$ (688.87): calcd $\mathrm{C} 76.72, \mathrm{H} \mathrm{7.02}$; found $\mathrm{C} 76.95, \mathrm{H} \mathrm{7.31}$; MS (FD): $688.2\left(\mathrm{M}^{+}\right), 344.2\left(\mathrm{M}^{2+}\right), 1376.1\left(2 \mathrm{M}^{+}\right)$.

Methyl-4-\{2,6-bis-[2-(2,5-dipropyloxy-4-\{2-[5-iod-3-(tetrahydro-2H-pyran-2yloxy)phenyl]ethynyl\}phenyl)ethynyl]-4-methyl $\}$-phenoxybutyrate (10): $\mathrm{Pd}\left(\mathrm{PPh}_{3}\right)_{2} \mathrm{Cl}_{2}(44 \mathrm{mg})$ and $\mathrm{CuI}(22 \mathrm{mg})$ were added to a solution of $9(6.24 \mathrm{~g}, 14.5$ $\mathrm{mmol})$ and $\mathbf{8}(1.53 \mathrm{~g}, 2.2 \mathrm{mmol})$ in triethylamine $(20 \mathrm{~mL})$, and the mixture was stirred for $18 \mathrm{~h}$ at $60{ }^{\circ} \mathrm{C}$. Workup was performed as described for compund 7. The oily crude product was chromatographed over silica gel with $\mathrm{CH}_{2} \mathrm{Cl}_{2}$ /petroleum ether (1:2) as the eluent to recover most of the excess of $9\left(4.67 \mathrm{~g} ; R_{\mathrm{f}}=0.77\right)$. Changing the eluent to $\mathrm{CH}_{2} \mathrm{Cl}_{2} /$ petroleum ether $(2: 1)$ yielded $\mathbf{1 0}\left(R_{\mathrm{f}}=0.30\right)$ as a slightly yellow solid $(2.32 \mathrm{~g}$, $81 \%$ ). M. p. $73{ }^{\circ} \mathrm{C} ;{ }^{1} \mathrm{H}$ NMR $\left(300 \mathrm{MHz}, \mathrm{CD}_{2} \mathrm{Cl}_{2}\right) \delta=7.52$ (t, $\left.J=1.4 \mathrm{~Hz}, 2 \mathrm{H}\right), 7.40-$ $7.43(\mathrm{~m}, 2 \mathrm{H}), 7.31(\mathrm{~s}, 2 \mathrm{H}), 7.18-7.22(\mathrm{~m}, 2 \mathrm{H}), 7.06(\mathrm{~s}, 2 \mathrm{H}), 7.03(\mathrm{~s}, 2 \mathrm{H}) 5.41(\mathrm{t}, J=$ $3.1 \mathrm{~Hz}, 2 \mathrm{H}), 4.39(\mathrm{t}, J=5.9 \mathrm{~Hz}, 2 \mathrm{H}), 4.02(\mathrm{t}, J=6.5 \mathrm{~Hz}, 4 \mathrm{H}), 4.01(\mathrm{t}, J=6.5 \mathrm{~Hz}, 4$ $\mathrm{H}), 3.80-3.90(\mathrm{~m}, 2 \mathrm{H}), 3.57-3.66(\mathrm{~m}, 2 \mathrm{H}), 3.55(\mathrm{~s}, 3 \mathrm{H}), 2.68(\mathrm{t}, J=7.4 \mathrm{~Hz}, 2 \mathrm{H})$, $2.32(\mathrm{~s}, 3 \mathrm{H}), 2.08-2.18(\mathrm{~m}, 2 \mathrm{H}), 1.50-2.05(\mathrm{~m}, 20 \mathrm{H}), 1.11(\mathrm{t}, J=7.3 \mathrm{~Hz}, 6 \mathrm{H}), 1.09$ $(\mathrm{t}, J=7.3 \mathrm{~Hz}, 6 \mathrm{H}) ;{ }^{13} \mathrm{C}-\mathrm{NMR}\left(75 \mathrm{MHz}, \mathrm{CD}_{2} \mathrm{Cl}_{2}\right): \delta=173.94,158.81,157.85,154.17$, $154.03,135.22,134.70,133.73,133.67,126.60,126.30,119.23,117.79,117.59$, $117.28,114.71,114.00,97.15,93.80,93.22,91.47,90.52,87.45,73.75,71.61,71.52$, $62.48,31.13,30.59,26.13,25.51,23.14,23.08,20.52,18.77,10.72,10.67$; elemental analysis for $\mathrm{C}_{66} \mathrm{H}_{70} \mathrm{I}_{2} \mathrm{O}_{11}$ (1293.10): calcd C 61.31, H 5.46; found $\mathrm{C}$ 61.56, H 5.54; MS (FD): $1292.3\left(\mathrm{M}^{+}\right), 646.1\left(\mathrm{M}^{2+}\right), 2586.7\left(2 \mathrm{M}^{+}\right)$.

Methyl-4-\{2,6-bis-[2-(2,5-dipropyloxy-4-\{2-[3-(tetrahydro-2H-pyran-2-yloxy)-5(2-trimethylsilylethynyl)phenyl]ethynyl phenyl)ethynyl]-4-methyl\}-

phenoxybutyrate (11): $\mathrm{Pd}\left(\mathrm{PPh}_{3}\right)_{2} \mathrm{Cl}_{2}(36 \mathrm{mg})$ and $\mathrm{CuI}(18 \mathrm{mg})$ were added to a solution of $10(2.15 \mathrm{~g}, 1.66 \mathrm{mmol})$ and TMS acetylene $(0.98 \mathrm{~g}, 9.96 \mathrm{mmol})$ in piperidine $(20 \mathrm{~mL})$, and the mixture was stirred for $16 \mathrm{~h}$ at $60{ }^{\circ} \mathrm{C}$. Workup was performed as described for compund 7. Column chromatography over silica gel with $\mathrm{CH}_{2} \mathrm{Cl}_{2}$ /petroleum ether $(2: 1)$ as the eluent $\left(R_{\mathrm{f}}=0.66\right)$ and subsequent radial 
chromatography over silica gel/gypsum with $\mathrm{CH}_{2} \mathrm{Cl}_{2} /$ petroleum ether (3:2) as the eluent gave 11 as a slightly yellow oil which slowly solidified (1.76 g, $86 \%$ ). M. p. $83{ }^{\circ} \mathrm{C} ;{ }^{1} \mathrm{H}$ NMR $\left(300 \mathrm{MHz}, \mathrm{CD}_{2} \mathrm{Cl}_{2}\right) \delta=7.31(\mathrm{~s}, 2 \mathrm{H}), 7.27(\mathrm{t}, J=1.3 \mathrm{~Hz}, 2 \mathrm{H}), 7.18$ $7.20(\mathrm{~m}, 2 \mathrm{H}), 7.11-7.13(\mathrm{~m}, 2 \mathrm{H}), 7.06(\mathrm{~s}, 2 \mathrm{H}), 7.03(\mathrm{~s}, 2 \mathrm{H}) 5.43(\mathrm{t}, \mathrm{J}=2.9 \mathrm{~Hz}, 2 \mathrm{H})$, $4.39(\mathrm{t}, J=5.9 \mathrm{~Hz}, 2 \mathrm{H}), 4.02(\mathrm{t}, J=6.5 \mathrm{~Hz}, 4 \mathrm{H}), 4.01$, (t, $J=6.5 \mathrm{~Hz}, 4 \mathrm{H}), 3.80-$ $3.90(\mathrm{~m}, 2 \mathrm{H}), 3.55-3.65(\mathrm{~m}, 2 \mathrm{H}), 3.55(\mathrm{~s}, 3 \mathrm{H}), 2.69$ (t, $J=7.6 \mathrm{~Hz}, 2 \mathrm{H}), 2.31(\mathrm{~s}, 3$ $\mathrm{H}), 2.08-2.18(\mathrm{~m}, 2 \mathrm{H}), 1.50-2.05(\mathrm{~m}, 20 \mathrm{H}), 1.12(\mathrm{t}, J=7.3 \mathrm{~Hz}, 6 \mathrm{H}), 1.09(\mathrm{t}, J=$ $7.3 \mathrm{~Hz}, 6 \mathrm{H}), \quad 0.26(\mathrm{~s}, 18 \mathrm{H}) ;{ }^{13} \mathrm{C}-\mathrm{NMR}\left(75 \mathrm{MHz}, \mathrm{CD}_{2} \mathrm{Cl}_{2}\right): \delta=173.96,158.84$, $157.35,154.19,154.07,134.70,133.67,128.59,125.00,124.84,120.41,120.32$, $117.85,117.66,117.37,114.61,114.28,104.37,97.05,95.20,94.16,91.44,90.60$, $86.83,73.76,71.66,71.55,62.47,51.59,31.17,30.68,26.19,25.59,23.19,23.12$, 20.57, 19.07, 10.76, 10.70, -0.03; elemental analysis for $\mathrm{C}_{76} \mathrm{H}_{88} \mathrm{O}_{11} \mathrm{Si}_{2}$ (1233.71): calcd C 73.99, H 7.19; found C 74.07, H 7.11; MS (FD): $1232.9\left(\mathrm{M}^{+}\right)$.

\section{Methyl-4-\{2,6-bis-[2-(2,5-dipropyloxy-4-\{2-[3-(tetrahydro-2H-pyran-2-yloxy)-5-} ethynylphenyl]ethynyl\}phenyl)ethynyl]-4-methyl\}-phenoxybutyrate (12): 11 (1.43 g; $1.16 \mathrm{mmol})$ and $\mathrm{K}_{2} \mathrm{CO}_{3}(0.81 \mathrm{~g}, 5.80 \mathrm{mmol})$ were stirred in $\mathrm{MeOH} / \mathrm{THF}(1: 1,40$ $\mathrm{mL}$ ) for $24 \mathrm{~h}$. The mixture was stirred for $2 \mathrm{~h}$ at room temperature and then poured into ether and water. The organic layer was extracted with water and brine and dried over $\mathrm{MgSO}_{4}$. After evaporation of the solvent the residue was purified by chromatography over silica gel with $\mathrm{CH}_{2} \mathrm{Cl}_{2}$ as the eluent $\left(R_{\mathrm{f}}=0.63\right)$ to give 12 as a slightly yellow solid (1.12 g, $89 \%)$. M.p. $>260{ }^{\circ} \mathrm{C} ;{ }^{1} \mathrm{H} \mathrm{NMR}\left(300 \mathrm{MHz}, \mathrm{CD}_{2} \mathrm{Cl}_{2}\right) \delta=$ $7.32(\mathrm{~s}, 2 \mathrm{H}), 7.29(\mathrm{t}, J=1.3 \mathrm{~Hz}, 2 \mathrm{H}), 7.22-7.25(\mathrm{~m}, 2 \mathrm{H}), 7.15-7.18(\mathrm{~m}, 2 \mathrm{H}), 7.06$ $(\mathrm{s}, 2 \mathrm{H}), 7.04(\mathrm{~s}, 2 \mathrm{H}) 5.43(\mathrm{t}, J=3.1 \mathrm{~Hz}, 2 \mathrm{H}), 4.39(\mathrm{t}, J=5.9 \mathrm{~Hz}, 2 \mathrm{H}), 4.02$ (t, $J=$ $6.5 \mathrm{~Hz}, 4 \mathrm{H}), 4.01(\mathrm{t}, J=6.5 \mathrm{~Hz}, 4 \mathrm{H}), 3.80-3.90(\mathrm{~m}, 2 \mathrm{H}), 3.55-3.65(\mathrm{~m}, 2 \mathrm{H}), 3.55$ $(\mathrm{s}, 3 \mathrm{H}), 3.16(\mathrm{~s}, 2 \mathrm{H}), 2.69$ (t, J=7.6 Hz, $2 \mathrm{H}), 2.31$ (s, $3 \mathrm{H}), 2.09-2.19(\mathrm{~m}, 2 \mathrm{H}), 1.55-$ $2.05(\mathrm{~m}, 20 \mathrm{H}), 1.12(\mathrm{t}, J=5.7 \mathrm{~Hz}, 6 \mathrm{H}), 1.09(\mathrm{t}, J=5.7 \mathrm{~Hz}, 6 \mathrm{H}) ;{ }^{13} \mathrm{C}-\mathrm{NMR}(75$ $\left.\mathrm{MHz}, \mathrm{CD}_{2} \mathrm{Cl}_{2}\right): \delta=173.95,158.82,157.37,154.17,154.05,134.69,133.66,128.68$, $125.08,123.74,120.80,120.52,117.82,117.58,117.30,114.61,114.15,97.11,94.02$, $91.44,90.57,86.92,83.00,77.91,73.74,71.61,71.50,62.50,51.60,31.15,30.64$, $26.16,25.55,23.17,23.09,20.54,19.08,10.76,10.69$; elemental analysis for $\mathrm{C}_{70} \mathrm{H}_{72} \mathrm{O}_{11}$ (1089.35): calcd C 77.18, H 6.66; found C 76.94, H 6.50; GPC (PS, THF): single peak with $M_{w}=1104, M_{n}=1084, P D=1.02$; MS (FD): $1088.7\left(\mathrm{M}^{+}\right)$.

THP-protected macrocycle 13: A solution of $12(485 \mathrm{mg}, 0.45 \mathrm{mmol})$ in pyridine $(18 \mathrm{~mL})$ was added to a suspension of $\mathrm{CuCl}(2.48 \mathrm{~g}, 26 \mathrm{mmol})$ and $\mathrm{CuCl}_{2}(0.49 \mathrm{~g}, 2.1$ mmol) in pyridine $(60 \mathrm{~mL})$ over $96 \mathrm{~h}$ at $60{ }^{\circ} \mathrm{C}$. After the completion of the addition, the mixture was allowed to stir for an additional $24 \mathrm{~h}$ at room temperature and then was poured into $\mathrm{CH}_{2} \mathrm{Cl}_{2}(500 \mathrm{~mL})$ and water $(200 \mathrm{~mL})$. The organic phase was extracted with water, $25 \% \mathrm{NH}_{3}$ solution (in order to remove the copper salts), water, $10 \%$ acetic acid, water, $10 \%$ aqueous sodium hydroxide, and brine, and dried over $\mathrm{MgSO}_{4}$. After evaporation of the solvent to a small volume and the coupling products were precipitated by the addition of methanol $(50 \mathrm{~mL})$ and collected by filtration. Repeated radial chromatography with $\mathrm{CH}_{2} \mathrm{Cl}_{2} /$ methanol (250:1) as the eluent gave 12 as a slightly yellow solid (140 mg; $29 \%)\left(R_{\mathrm{f}}\left(\mathrm{CH}_{2} \mathrm{Cl}_{2}\right)=0.48\right)$. M. p. $>240{ }^{\circ} \mathrm{C} ;{ }^{1} \mathrm{H}-$ NMR (300 MHz, $\mathrm{CD}_{2} \mathrm{Cl}_{2}$ ): $\delta=7.37(\mathrm{t}, J=1.4 \mathrm{~Hz}, 4 \mathrm{H}), 7.29-7.31(\mathrm{~m}, 4 \mathrm{H}), 7.23-7.26$ (m, 4 H), 7.17-7.20 (m, 4 H), $7.06(\mathrm{~s}, 4 \mathrm{H}), 7.05(\mathrm{~s}, 4 \mathrm{H}) 5.44(\mathrm{t}, J=2.9 \mathrm{~Hz}, 4 \mathrm{H}), 4.38$ $(\mathrm{t}, J=5.9 \mathrm{~Hz}, 4 \mathrm{H}), 4.03(\mathrm{t}, J=6.5 \mathrm{~Hz}, 8 \mathrm{H}), 4.02(\mathrm{t}, J=6.5 \mathrm{~Hz}, 8 \mathrm{H}), 3.82-3.92(\mathrm{~m}, 4$ $\mathrm{H}), 3.55-3.66(\mathrm{~m}, 4 \mathrm{H}), 3.56(\mathrm{~s}, 6 \mathrm{H}), 2.68(\mathrm{t}, J=7.4 \mathrm{~Hz}, 4 \mathrm{H}), 2.32(\mathrm{~s}, 6 \mathrm{H}), 2.06-2.26$ 
$(\mathrm{m}, 4 \mathrm{H}), 1.55-2.05(\mathrm{~m}, 40 \mathrm{H}), 1.13(\mathrm{t}, J=7.6 \mathrm{~Hz}, 4 \mathrm{H}), 1.10(\mathrm{t}, J=7.6 \mathrm{~Hz}, 4 \mathrm{H})$; elemental analysis for $\mathrm{C}_{140} \mathrm{H}_{140} \mathrm{O}_{22}$ (2174.66): calcd C 77.33, H 6.49; found $\mathrm{C} 77.02$, H 6.68; GPC (PS, THF): single peak with $M_{w}=1943, M_{n}=1908, \mathrm{PD}=1.02$.

Macrocycle 14: To a suspension of $13(300 \mathrm{mg}, 0.14 \mathrm{mmol})$ in $\mathrm{CHCl}_{3}(120 \mathrm{~mL})$ was slowly added $\mathrm{MeOH}(9 \mathrm{~mL})$ and then $p$-toluenesulfonic acid (10-15 mg). The mixture was stirred for $3 \mathrm{~d}$ at room temperature under an argon atmosphere. The mixture was evaporated to a small volume and methanol $(200 \mathrm{~mL})$ was added, and the yellow precipitate was collected by filtration and vacuum dried in the dark to give of $\mathbf{1 4}$ as a slightly yellow solid (210 mg; $68 \%$ ): M. p. > $260{ }^{\circ} \mathrm{C} ;{ }^{1} \mathrm{H}-\mathrm{NMR}\left(300 \mathrm{MHz}, \mathrm{THF}-\mathrm{d}_{8}\right)$ : $\delta=9.01(\mathrm{~s}, 4 \mathrm{H}), 7.28(\mathrm{~s}, 4 \mathrm{H}), 7.20-7.22(\mathrm{~m}, 4 \mathrm{H}), 7.11(\mathrm{~s}, 4 \mathrm{H}), 7.10(\mathrm{~s}, 4 \mathrm{H}), 6.93-$ $6.96(\mathrm{~m}, 4 \mathrm{H}), 6.86-6.92(\mathrm{~m}, 4 \mathrm{H}), 4.39(\mathrm{t}, J=5.9 \mathrm{~Hz}, 4 \mathrm{H}), 4.05(\mathrm{t}, J=6.5 \mathrm{~Hz}, 8 \mathrm{H})$, $4.04(\mathrm{t}, J=6.5 \mathrm{~Hz}, 8 \mathrm{H}) 3.55(\mathrm{~s}, 6 \mathrm{H}), 2.70(\mathrm{t}, J=7.6 \mathrm{~Hz}, 4 \mathrm{H}), 2.30(\mathrm{~s}, 6 \mathrm{H}), 2.10-2.25$ $(\mathrm{m}, 4 \mathrm{H}), 1.75-2.00(\mathrm{~m}, 16 \mathrm{H}), 1.14(\mathrm{t}, J=7.3 \mathrm{~Hz}, 12 \mathrm{H}), 1.10(\mathrm{t}, J=7.3 \mathrm{~Hz}, 12 \mathrm{H})$; ${ }^{13} \mathrm{C}-\mathrm{NMR}\left(75 \mathrm{MHz}, \mathrm{THF}-\mathrm{d}_{8}\right): \delta=173.78,158.75,154.90,154.66,134.52,133.71$, 128.14, 126.26, 123.68, 120.06, 119.39, 118.68, 117.86, 115.41, 114.75, 94.21, 91.71, $91.17,87.52,81.62,74.29,74.06,71.81,71.74,51.36,31.36,30.62,26.80,23.66$, 23.58, 20.38, 10.97, 10.81; elemental analysis for $\mathrm{C}_{120} \mathrm{H}_{108} \mathrm{O}_{18}$ (1383.19): calcd $\mathrm{C}$ 78.41, H 5.92; found C 78.19, H 5.53; MS (FD): $1383.6\left(\mathrm{M}^{+}\right)$.

Macrocycle 3a: $\mathrm{K}_{2} \mathrm{CO}_{3}(250 \mathrm{mg} ; 1.81 \mathrm{mmol})$ was added to a solution of 14 (70 $\mathrm{mg}$, $50 \mu \mathrm{mol})$ and 3,4,5-tris-(dodecyloxy)-benzyl chloride (150 mg, $0.22 \mathrm{mmol}$ ) in DMF $(80 \mathrm{~mL})$ and stirred for $3 \mathrm{~d}$ at $60^{\circ} \mathrm{C}$ in the dark. After the reaction mixture cooled down to room temperature, $\mathrm{CH}_{2} \mathrm{Cl}_{2}$ and water were added. The organic phase was separated and extracted with water and brine, and dried over $\mathrm{MgSO}_{4}$. After evaporation of most of the solvent methanol $(100 \mathrm{~mL})$ were added and the precipitate collected by filtration. The crude product was purified by radial chromatography over silica gel/gypsum with $\mathrm{CH}_{2} \mathrm{Cl}_{2}$ as the eluent $\left(R_{\mathrm{f}}=0.77\right)$ to give $\mathbf{3 a}$ as a slightly yellow solid (64 mg, $29 \%$ ): $\mathrm{mp} 102{ }^{\circ} \mathrm{C}$ (DSC, first heating); ${ }^{1} \mathrm{H}-\mathrm{NMR}\left(250 \mathrm{MHz}, \mathrm{CD}_{2} \mathrm{Cl}_{2}\right): \delta$ $=7.37(\mathrm{t}, J=1.2 \mathrm{~Hz}, 4 \mathrm{H}), 7.30(\mathrm{~s}, 4 \mathrm{H}), 7.15-7.17(\mathrm{~m}, 4 \mathrm{H}), 7.08-7.12(\mathrm{~m}, 4 \mathrm{H}), 7.07$ $(\mathrm{s}, 4 \mathrm{H}), 7.05(\mathrm{~s}, 4 \mathrm{H}), 6.63(\mathrm{~s}, 8 \mathrm{H}) 4.98(\mathrm{~s}, 8 \mathrm{H}), 4.38(\mathrm{t}, J=6.0 \mathrm{~Hz}, 4 \mathrm{H}), 3.88-4.06$ $(\mathrm{m}, 40 \mathrm{H}), 3.56(\mathrm{~s}, 6 \mathrm{H}), 2.69(\mathrm{t}, J=7.5 \mathrm{~Hz}, 4 \mathrm{H}), 2.32(\mathrm{~s}, 6 \mathrm{H}), 2.08-2.22(\mathrm{~m}, 4 \mathrm{H})$, $1.65-1.98(\mathrm{~m}, 40 \mathrm{H}), 1.20-1.54(\mathrm{~m}, 216 \mathrm{H}), 1.13(\mathrm{t}, J=7.3 \mathrm{~Hz}, 12 \mathrm{H}), 1.10(\mathrm{t}, J=$ $7.3 \mathrm{~Hz}, 4 \mathrm{H}), 0.80-0.95(\mathrm{~m}, 36 \mathrm{H}) ;{ }^{13} \mathrm{C}-\mathrm{NMR}\left(75 \mathrm{MHz}, \mathrm{CD}_{2} \mathrm{Cl}_{2}\right): \delta=159.16,154.31$, $154.06,153.82,138.54,134.43,133.74,131.65,129.41,125.56,123.33,117.90$, $117.64,117.46,114.88,114.01,106.53,93.74,91.45,90.58,87.40,81.32,74.37$, $73.80,71.65,71.20,69.60,32.37,31.19,30.81$, several peaks between 29.5 and 30.5 ppm, 26.57, 26.22, 23.21, 23.11, 14.27, 10.77, 10.68; elemental analysis for $\mathrm{C}_{293} \mathrm{H}_{424} \mathrm{O}_{30}$ (4426.63): calcd C 79.50, H 9.65; found C 79.72, H 9.48; GPC (PS, THF): single peak with $M_{w}=4829, M_{n}=4776, \mathrm{PD}=1.02$; MS (MALDI, $\mathrm{Ag}^{+}$): 4519 $\left(\mathrm{M}+\mathrm{Ag}^{+}\right)$.

Macrocycle 3b: Compound 3b was prepared by the procedure described for $1 \mathbf{a}$, by adding $\mathrm{K}_{2} \mathrm{CO}_{3}(2.0 \mathrm{~g} ; 14.8 \mathrm{mmol})$ to a solution of $14(80 \mathrm{mg}, 40 \mu \mathrm{mol})$ and 3,4,5-tris(hexadecyloxy)-benzyl chloride $(320 \mathrm{mg}, 0.38 \mathrm{mmol})$ in $\mathrm{DMF} / \mathrm{CH}_{3} \mathrm{Cl}(10: 1)(550$ $\mathrm{mL})$. Purification was performed by repeated radial chromatography over silica gel/gypsum first with $\mathrm{CH}_{2} \mathrm{Cl}_{2} /$ methanol $(250: 1)$ as the eluent $\left(R_{\mathrm{f}}=0.99\right)$ and then using a gradient $\mathrm{CH}_{2} \mathrm{Cl}_{2} /$ petroleum ether $(1: 1)$ to $\mathrm{CH}_{2} \mathrm{Cl}_{2}$ as the eluent $\left(R_{\mathrm{f}}\left(\mathrm{CH}_{2} \mathrm{Cl}_{2}\right)=\right.$ $0.93)$ to give $\mathbf{3 b}(99 \mathrm{mg}, 49 \%)$ as slightly yellow solid. M. p. $92{ }^{\circ} \mathrm{C}$ (DSC, first heating); $\delta=7.35-7.40(\mathrm{~m}, 4 \mathrm{H}), 7.30(\mathrm{~s}, 4 \mathrm{H}), 7.15-7.18(\mathrm{~m}, 4 \mathrm{H}), 7.08-7.13$ (m, 4 
H), $7.06(\mathrm{~s}, 4 \mathrm{H}), 7.04(\mathrm{~s}, 4 \mathrm{H}), 6.63(\mathrm{~s}, 8 \mathrm{H}) 4.98(\mathrm{~s}, 8 \mathrm{H}), 4.38(\mathrm{t}, J=5.7 \mathrm{~Hz}, 4 \mathrm{H})$, 3.88-4.08 (m, $40 \mathrm{H}), 3.55(\mathrm{~s}, 6 \mathrm{H}), 2.68(\mathrm{t}, J=7.5 \mathrm{~Hz}, 4 \mathrm{H}), 2.32(\mathrm{~s}, 6 \mathrm{H}), 2.08-2.22$ (m, $4 \mathrm{H}), 1.65-1.98(\mathrm{~m}, 40 \mathrm{H}), 1.20-1.54(\mathrm{~m}, 312 \mathrm{H}), 1.13(\mathrm{t}, J=7.3 \mathrm{~Hz}, 12 \mathrm{H}), 1.09$ $(\mathrm{t}, J=7.3 \mathrm{~Hz}, 4 \mathrm{H}), 0.80-0.95(\mathrm{~m}, 36 \mathrm{H}) ; ;{ }^{13} \mathrm{C}-\mathrm{NMR}\left(75 \mathrm{MHz}, \mathrm{CD}_{2} \mathrm{Cl}_{2}\right): \delta=173.93$, $161.10,159.10,154.23,153.99,153.77,153.66,138.52,138.37,134.42,133.72$, $131.61,130.81,125.52,123.28,117.86,117.48,117.30,114.76,113.89,107.02$, $106.37,93.72,91.43,90.55,87.37,81.29,74.35,73.76,71.52,71.14,69.47,51.62$, 32.36, several peaks between 29.5 and 31 ppm, 26.56, 23.12, 14.29, 10.98, 10.78; elemental analysis for $\mathrm{C}_{340} \mathrm{H}_{516} \mathrm{O}_{30}(5083.83)$ : calcd $\mathrm{C} 80.33, \mathrm{H} \mathrm{10.23}$; found $\mathrm{C} 80.07$, H 9.94; GPC (PS, THF): single peak with $M_{w}=8146, M_{n}=7982, \mathrm{PD}=1.02$; $\mathrm{MS}$ (MALDI): $5191.8\left(\mathrm{M}+\mathrm{Ag}^{+}\right)$or $5125\left(\mathrm{M}+\mathrm{K}^{+}\right)$.

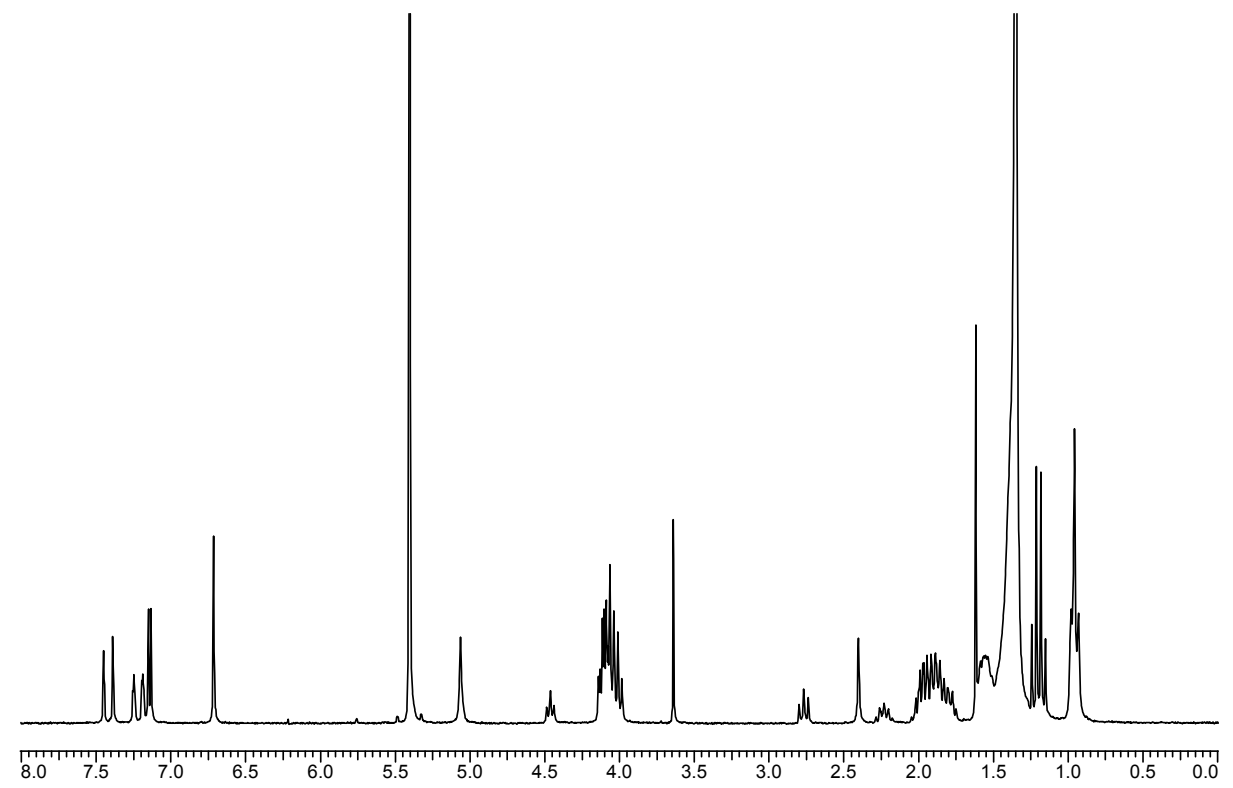

Figure 1. 1H NMR of 3a. 


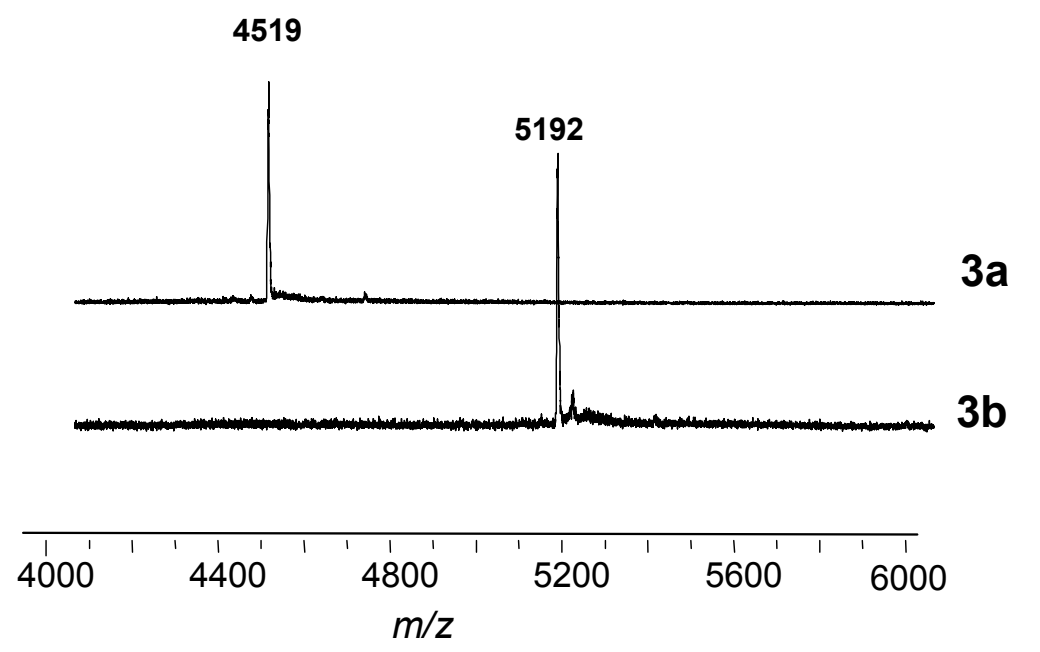

Figure 2. MALDI-TOF spectra of $\mathbf{3 a}$ and $\mathbf{3 b}$.



Figure 3. Electron diffraction pattern of a thin film of $\mathbf{3 b}$, recorded at room temperature showing a ring, corresponding to a $d$-value of $4.1 \AA$. 
STM images of monolayers of $\mathbf{3 a}$ (Figure $4 a, b)$ and $\mathbf{3 b}$ (Figure $4 \mathrm{c}, \mathrm{d}$ ) at the liquid (1,2,4-trichlorobenzene) - solid (graphite) interface. The images show less resolution than those obtained at the liquid (1-phenyloctane) - solid (graphite) interface, although often long-range ordering can be observed.

a)

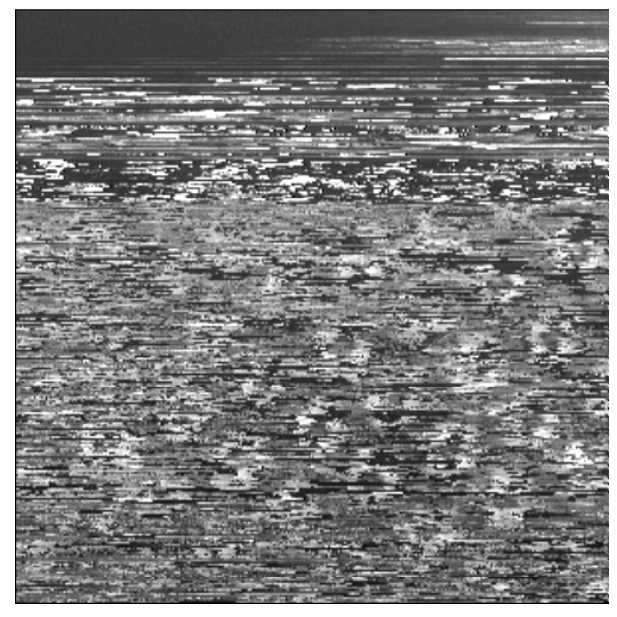

c)

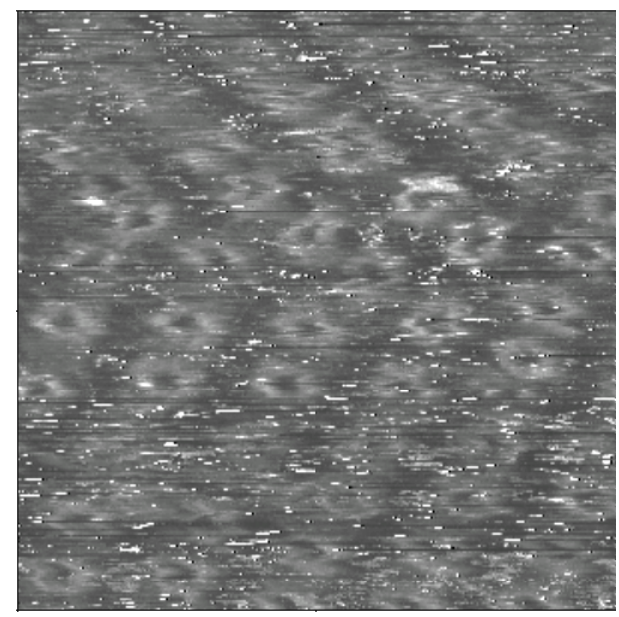

b)

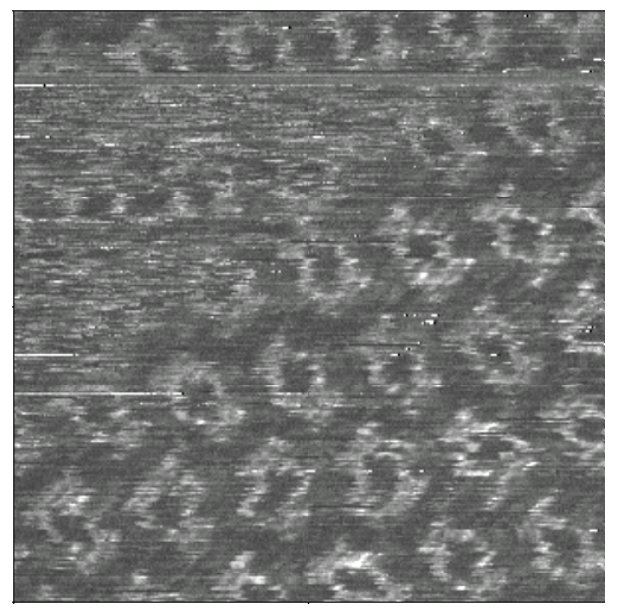

d)

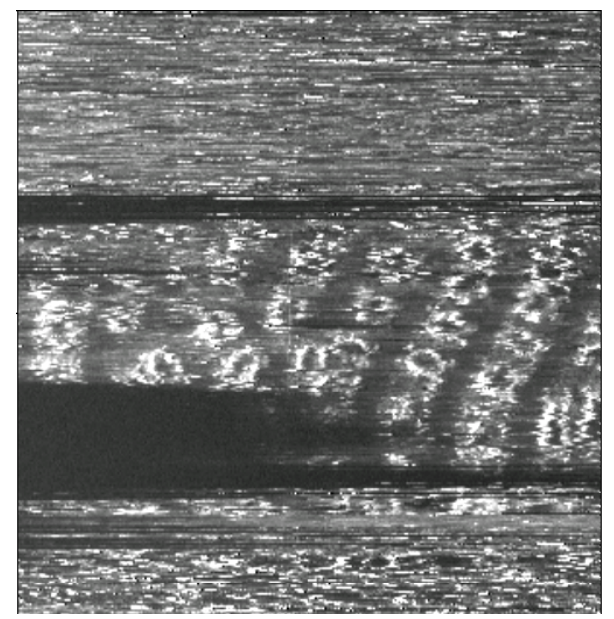

Figure 4. $(\mathrm{a}, \mathrm{b})$ : STM images of $\mathbf{3 a}$ at the liquid (1,2,4-trichlorobenzene) - solid (graphite) interface: (a) $33.25 \times 33.25 \mathrm{~nm}^{2}, \mathrm{I}=0.75 \mathrm{nA}$; V = -0.768 V; (b) $22.6 \times 22.6$ $\mathrm{nm}^{2}, \mathrm{I}=0.7 \mathrm{nA} ; \mathrm{V}=-0.832 \mathrm{~V}$. (c, d): STM images of $\mathbf{3 b}$ at the liquid $(1,2,4-$ trichlorobenzene) - solid (graphite) interface: (c) $32.38 \times 32.38 \mathrm{~nm}^{2}, \mathrm{I}=0.65 \mathrm{nA}$; V = $-1.124 \mathrm{~V}$; (d) $46 \times 46 \mathrm{~nm}^{2}, \mathrm{I}=0.45 \mathrm{nA} ; \mathrm{V}=-1.562 \mathrm{~V}$. 